\title{
Simulation of dynamics-coupling in piezoelectric tube scanners by reduced order finite element analysis
}

\author{
Johannes Maess ${ }^{\text {a) }}$ \\ Institute for Systems Theory and Automatic Control, University of Stuttgart, Pfaffenwaldring 9, \\ 70550 Stuttgart, Germany \\ Andrew J. Fleming ${ }^{\text {b) }}$ \\ School of Electrical Engineering and Computer Science, The University of Newcastle, University Drive, \\ Callaghan, New South Wales 2308, Australia \\ Frank Allgöwer ${ }^{\mathrm{c})}$ \\ Institute for Systems Theory and Automatic Control, University of Stuttgart, Pfaffenwaldring 9, \\ 70550 Stuttgart, Germany
}

(Received 8 August 2007; accepted 28 November 2007; published online 11 January 2008)

\begin{abstract}
Piezoelectric tube scanners are widely used in scanning probe microscopes to position the sample or the probe. Fast and accurate scanning requires the suppression of dominant low-frequency resonances as well as the compensation of dynamics-coupling effects. The present article gives a detailed description of the fully coupled tube scanner dynamics over a wide frequency range modeled by finite element (FE) analysis using the commercially available software package ANSYS. The effect of a sample mass attached to the top of the tube is investigated by considering its added mass and local stiffening. A model order reduction scheme is applied to obtain a low order model that describes the lateral and vertical deflections as well as the voltage induced on quadrant electrodes. Comparison to experimental data demonstrates a good agreement for both the full FE model and reduced order model. (C) 2008 American Institute of Physics. [DOI: 10.1063/1.2826428]
\end{abstract}

\section{INTRODUCTION}

In the field of nanopositioning, piezoelectric tube scanners are widespread due to their capability for threedimensional positioning with high resolution and speed. They are simple to manufacture and are readily available at low cost. ${ }^{1}$ Since their invention, ${ }^{2}$ the most common application of piezoelectric tube scanners has been in positioning stages for scanning probe microscopes.

The tube scanner apparatus is illustrated in Fig. 1. The base of the tube is glued to a mounting fixture and a sample holder is bonded to the top surface. Four quartered electrodes are deposited onto the outer diameter of the piezoceramic material, whereas the inner surface of the tube is coated with a single electrode. During operation, one of the two outer $x$-axis electrodes is driven with a voltage for the generation of the lateral $x$-displacement by means of the indirect piezoelectric effect. This configuration is commonly referred to as antisymmetric excitation or single electrode excitation since only one electrode of a pair is driven. The remaining $x$-axis electrode can be used as a deflection sensor for feedback control purposes. The induced voltage on the sensor electrode is generated by the direct piezoelectric effect. In the setup under consideration one of the two $y$-axis electrodes is grounded while the other serves as sensor electrode for $y$-axis dynamics.

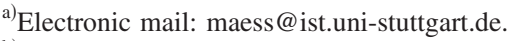

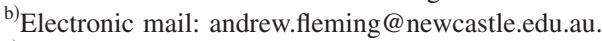

${ }^{c}$ Electronic mail: allgower@ist.uni-stuttgart.de.
}

Scanning accuracy is limited by a number of undesirable effects, the foremost of which are hysteresis, ${ }^{3}$ creep, ${ }^{4}$ coupling between the directions of motion, ${ }^{5}$ and induced vibrations. ${ }^{6}$ The present work focuses on the modeling and simulation of dynamics describing the two latter deficiencies. Complementary to previous work, ${ }^{7}$ the simulation model takes into account the local stiffening and added mass of the sample holder. The finite element (FE) model obtained by the use of ANSYS (Ref. 20) considers the piezoelectric tube as well as the sample mass as flexible bodies perfectly glued to each other with an appropriate coupling between the structural and electrical fields. Furthermore, the influence of machining tolerances and imperfections during the manufacturing process of the tube on the dynamics-coupling-caused errors in open-loop operation are investigated by simulation studies. In this respect, two typical deviations from the nominal tube considered in the FE model are the nonconcentricity of the inner and outer diameters ${ }^{8}$ and changes in the radial positions or size of the outer electrodes. The coupled-field finite element analysis (FEA) programed together with the model order reduction ${ }^{9}$ applied in this article provides an accurate description of all these effects in a single model.

Much effort has been spent on the improvement of positioning accuracy by means of feedforward ${ }^{10,11}$ and feedback control strategies. ${ }^{12}$ One limiting factor associated with feedback control is the requirement for a displacement signal that adds significant cost and complexity to a microscope. This difficulty is partially circumvented by employing the single electrode excitation setup with two sensor electrodes for estimation of the tip displacement in the $x$ - and 


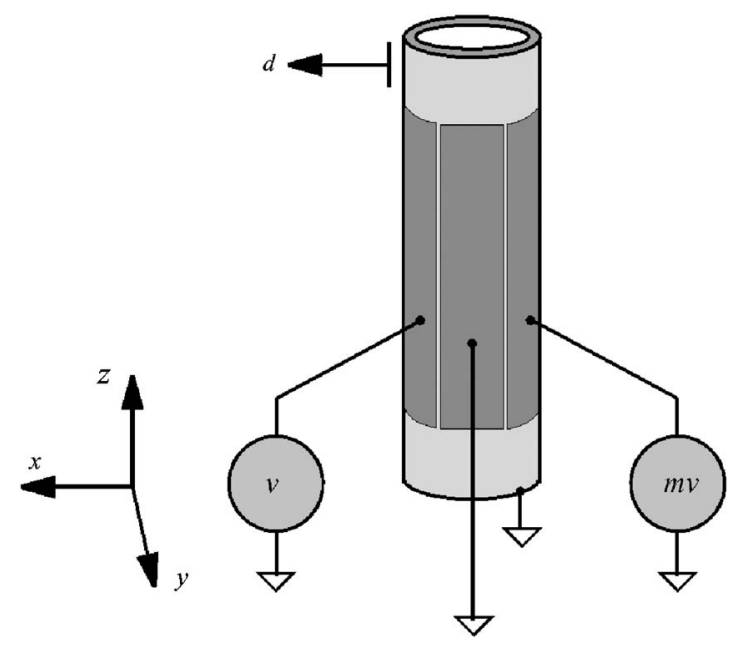

FIG. 1. Setup of the piezoelectric tube scanner, where $v$ is the applied voltage and $m v$ is the measured voltage.

$y$-directions. While this is investigated experimentally in Ref. 13, the present paper introduces a modeling approach using coupled-field FEA and the double piezoelectric effect.

\section{MODELING}

The FE method is used to obtain a very general and accurate model of the piezoelectric tube scanner. For the purpose of controller design and transient simulations, the high order of the full FE model is effectively reduced in the desired frequency range by model order reduction. This is based on modal truncation and consideration of the Hankel singular values of the desired input-output behavior. All equations are stated in their general form, thus being valid for the nominal tube scanner with or without sample mass as well as for modeled imperfections, demonstrating the flexibility of the approach.

\section{A. Finite element method}

Three-dimensional FEA is used in a variety of applications for the modeling of piezoceramic actuators and sensors, e.g., beam actuators ${ }^{14}$ and smart structures. ${ }^{15}$ FE models of piezoelectric tube scanners have so far only been used to describe the resulting static deflections on application of a driving voltage. ${ }^{16,17}$ Due to the modeling complexity, the scanner dynamics are typically identified from experimental data. ${ }^{6,18}$ In the present work, FEA is extended to gain a deeper mechanical understanding of the case where a sample holder is glued onto the scanner top. FEA also allows the prediction of the influence of changing tube dimensions and machining tolerances on the tube motion.

The constitutive equations for the fully coupled threedimensional piezoelectric material consisting of the direct and indirect piezoelectric effects are given by ${ }^{19}$

$$
\begin{aligned}
& \mathbf{T}=\mathbf{c}^{\mathbf{E}} \mathbf{S}-\mathbf{e E}, \\
& \mathbf{D}=\mathbf{e} \mathbf{S}+\boldsymbol{\epsilon}^{\mathbf{S}} \mathbf{E},
\end{aligned}
$$

TABLE I. Material properties of PZT-5H.

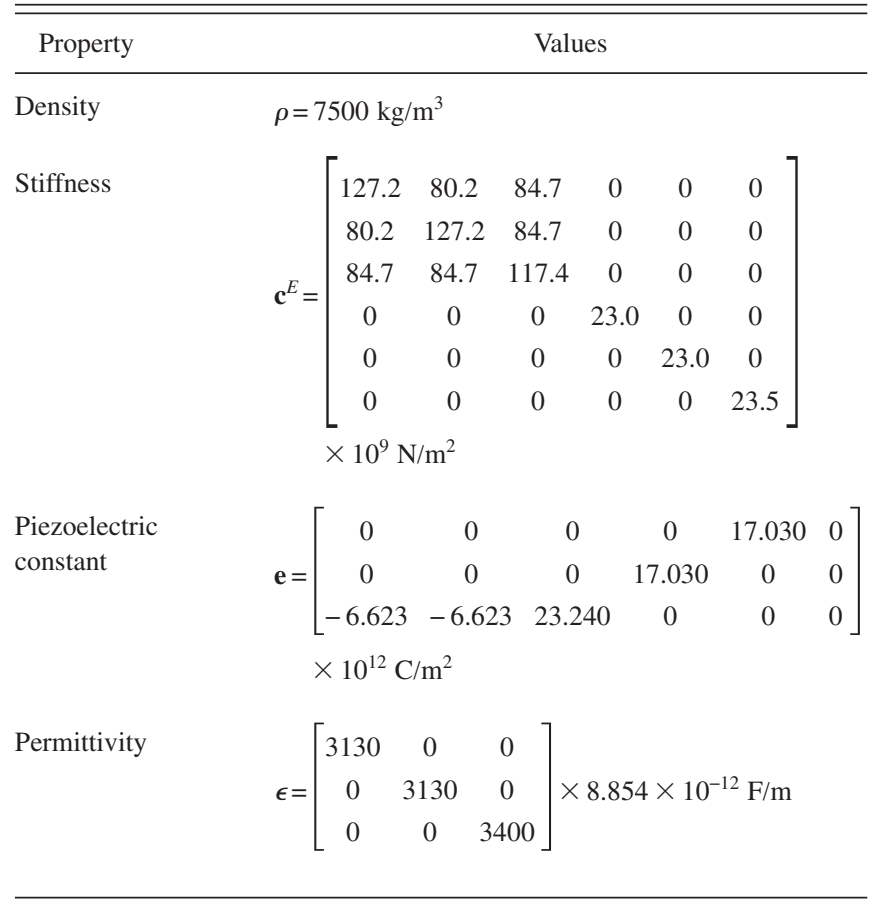

with mechanical stress matrix $\mathbf{T}$, mechanical strain matrix $\mathbf{S}$, electric field vector $\mathbf{E}$, and electric charge vector per unit area $\mathbf{D}$. The anisotropic material properties of the radially polarized piezoceramic are described by the mechanical stiffness matrix at constant electric field $\mathbf{c}^{\mathbf{E}}$, the permittivity matrix under constant strain $\boldsymbol{\epsilon}^{\mathbf{S}}$, and the piezoelectric stress matrix e. Assembly of the element matrices leads to the equations of motion in the general FE formulation,

$$
\mathbf{M} \ddot{\mathbf{x}}+\mathbf{C} \dot{\mathbf{x}}+\mathbf{K x}=\mathbf{F},
$$

with mass matrix $\mathbf{M}$, stiffness matrix $\mathbf{K}$, and external loads F. For simplicity, Rayleigh damping is assumed for the FE model via the damping matrix $\mathbf{C}$. The state vector $\mathbf{x}$ is composed of the nodal displacements $\mathbf{u}$ and the nodal electric potentials $\boldsymbol{\phi}$ accounting for the coupling between the structural and piezoelectric domains. The order of the fully coupled finite element model is denoted as $n$. The general form of Eq. (1) is not changed by considering the sample mass, which is assumed to be perfectly glued onto the tube and thus only adds degrees of freedom in the structural domain in form of the nodal displacements $\mathbf{u}$.

The FE discretized model of the pietzotube is constructed with the commercial software ANSYS (Ref. 20) using three-dimensional solid piezoelectric elements. The material properties of the piezoceramic PZT-5H used in the simulations are given in Table I. One benefit of the FEA is the classification of the eigenmodes of the piezoelectric tube scanner by means of the three-dimensional mode shapes as illustrated in Fig. 2 for the case without attached sample mass. From this, an overview of the scanner dynamics for a sufficiently fine meshed FE model stated in Table II is deduced. It can be observed that the low-frequency motion is dominated by longitudinal Bernoulli beam type bending 

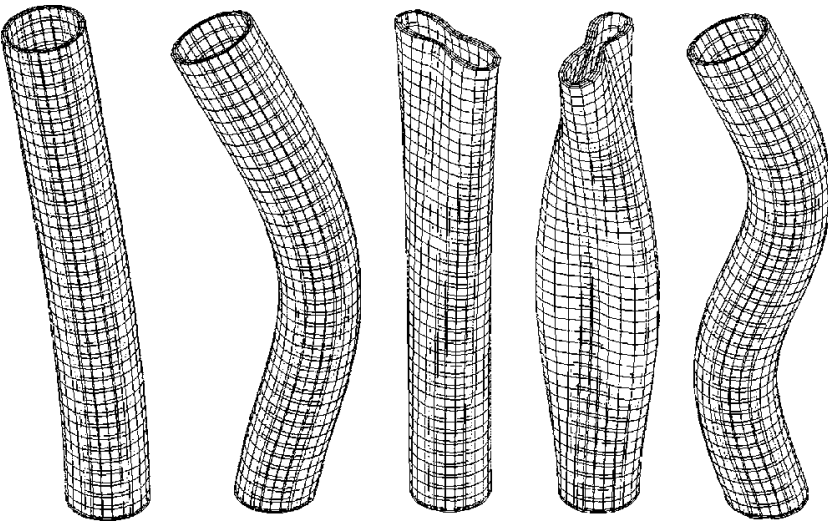

FIG. 2. Three-dimensional (3D) finite element mode shapes; from left to right: first longitudinal bending mode, second longitudinal bending mode, first circumferential bending mode, second circumferential bending mode, and third longitudinal bending mode.

modes. At higher frequencies, additional types of mode shapes, such as circumferential shell bending modes, torsional modes, and longitudinal extension, occur. All longitudinal and circumferential bending modes appear twice due to the axissymmetric geometry of the tube.

Table II further summarizes the change of eigenfrequencies when the sample mass is attached to the top of the tube. The sample holder consists of a hollow aluminum cube with a cylindrical extension for concentric placement on the tube scanner and is glued onto the free end of the tube. Its overall weight is $2.01 \mathrm{~g}$. As before, the simulated eigenfrequencies accurately match the measurement results. It can be seen that the longitudinal bending modes shown in Fig. 3 as well as the torsional and longitudinal compression modes are shifted to considerably lower eigenfrequencies due to the added mass of the sample holder. In addition, the circumferential bending modes which show large deformations towards the free end of the tube without sample mass do not occur any more, since the local stiffening of the sample holder glued to the tube top suppresses this deflection shape. Instead, circumferential bending modes appear now at high frequencies, with the mode shapes in Fig. 3 being similar to a tube clamped at both ends. In summary, the sample holder leads to a stiffening of the circumferential bending modes whereas
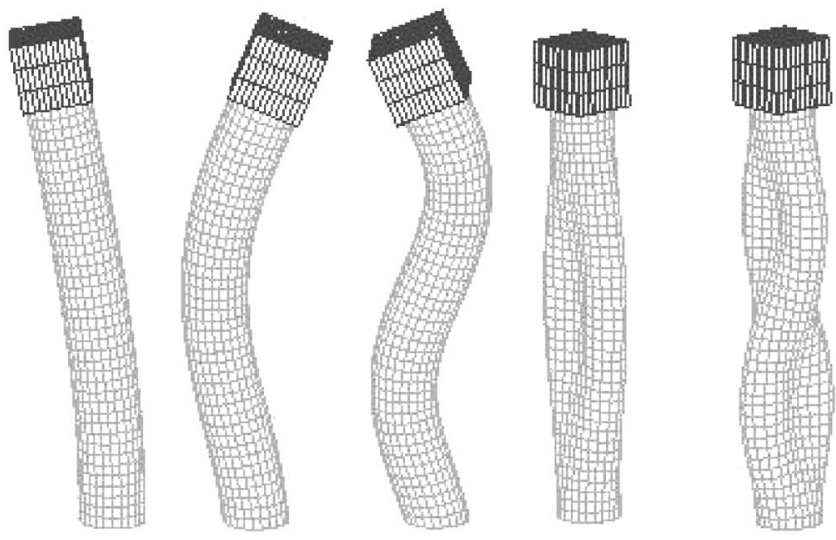

FIG. 3. 3D finite element mode shapes with sample mass; from left to right: first longitudinal bending mode, second longitudinal bending mode, third longitudinal bending mode, first circumferential bending mode, and second circumferential bending mode.

the longitudinal bending modes and the longitudinal extension modes are subject to softening effects.

The mesh density required for achieving accurate simulation results leads to a model order of $n \approx 10000$ in the full FE model of Eq. (1). This high order precludes the full model from controller design and transient simulations. In the following section, a model order reduction process offering high flexibility with respect to the bandwidth of the reduced model as well as its number of inputs and outputs is introduced.

\section{B. Model order reduction}

A detailed description of the model order reduction, which is carried out in MATLAB after importing the FE model from ANSYS, is given in Ref. 21. In the self-sensing configuration, the potential electrodes are driven by external voltages while the sensor electrodes, which are located pairwise opposite to the respective potential electrodes, are used to measure the resulting voltage feedback signal. Partitioning of the electrical degrees of freedom in the equations of motion of the full FE model in Eq. (1) into degrees of freedom on the grounded electrodes $\boldsymbol{\phi}_{g}$, on the potential electrodes $\boldsymbol{\phi}_{p}$, on the sensor electrodes $\boldsymbol{\phi}_{m}$, and in the interior of the piezotube $\boldsymbol{\phi}_{i}$ (Ref. 22) yields

TABLE II. Summary of the full FE modal analysis.

\begin{tabular}{|c|c|c|c|c|c|c|}
\hline \multirow[b]{2}{*}{ No. } & \multicolumn{3}{|c|}{ Eigenfrequency without sample mass $(\mathrm{kHz})$} & \multicolumn{3}{|c|}{ Eigenfrequency with sample mass $(\mathrm{kHz})$} \\
\hline & $\mathrm{FE}$ & Expt. & Classification & FE & Expt. & Classification \\
\hline 1,2 & 1.21 & 1.22 & First longitudinal bending & 0.83 & 0.84 & First longitudinal bending \\
\hline 3,4 & 6.49 & 6.55 & Second longitudinal bending & 4.89 & 4.84 & Second longitudinal bending \\
\hline 5 & 6.97 & $\cdots$ & Torsion & 5.72 & $\cdots$ & Torsion \\
\hline 6 & 11.30 & 11.30 & Longitudinal extension & 9.27 & 9.43 & Longitudinal extension \\
\hline 7,8 & 13.98 & 14.24 & First circumferential bending & 12.28 & 12.14 & Third longitudinal bending \\
\hline 9,10 & 14.50 & 15.21 & Second circumferential bending & 14.73 & 15.19 & First circumferential bending \\
\hline 11,12 & 15.38 & 15.79 & Third longitudinal bending & 16.30 & 16.68 & Second circumferential bending \\
\hline
\end{tabular}




$$
\left[\begin{array}{ccccc}
\mathbf{M}_{s s} & \mathbf{0} & \mathbf{0} & \mathbf{0} & \mathbf{0} \\
\mathbf{0} & \mathbf{0} & \mathbf{0} & \mathbf{0} & \mathbf{0} \\
\mathbf{0} & \mathbf{0} & \mathbf{0} & \mathbf{0} & \mathbf{0} \\
\mathbf{0} & \mathbf{0} & \mathbf{0} & \mathbf{0} & \mathbf{0} \\
\mathbf{0} & \mathbf{0} & \mathbf{0} & \mathbf{0} & \mathbf{0}
\end{array}\right]\left[\begin{array}{c}
\ddot{\mathbf{u}} \\
\ddot{\boldsymbol{\phi}}_{i} \\
\ddot{\boldsymbol{\phi}}_{m} \\
\ddot{\boldsymbol{\phi}}_{p} \\
\ddot{\boldsymbol{\phi}}_{g}
\end{array}\right]+\left[\begin{array}{ccccc}
\mathbf{K}_{s s} & \mathbf{K}_{s i} & \mathbf{K}_{s m} & \mathbf{K}_{s p} & \mathbf{K}_{s g} \\
\mathbf{K}_{s i}^{T} & \mathbf{K}_{i i} & \mathbf{K}_{i m} & \mathbf{K}_{i p} & \mathbf{K}_{i g} \\
\mathbf{K}_{s m}^{T} & \mathbf{K}_{i m}^{T} & \mathbf{K}_{m m} & \mathbf{K}_{m p} & \mathbf{K}_{m g} \\
\mathbf{K}_{s p}^{T} & \mathbf{K}_{i p}^{T} & \mathbf{K}_{m p}^{T} & \mathbf{K}_{p p} & \mathbf{K}_{p g} \\
\mathbf{K}_{s g}^{T} & \mathbf{K}_{i g}^{T} & \mathbf{K}_{m g}^{T} & \mathbf{K}_{p g}^{T} & \mathbf{K}_{g g}
\end{array}\right]\left[\begin{array}{c}
\mathbf{u} \\
\boldsymbol{\phi}_{i} \\
\boldsymbol{\phi}_{m} \\
\boldsymbol{\phi}_{p} \\
\boldsymbol{\phi}_{g}
\end{array}\right]=\left[\begin{array}{c}
\mathbf{f} \\
\mathbf{q}_{i} \\
\mathbf{q}_{m} \\
\mathbf{q}_{p} \\
\mathbf{q}_{g}
\end{array}\right],
$$

with the electrical charges $\mathbf{q}_{p}, \mathbf{q}_{g}$, and $\mathbf{q}_{m}$ on the potential, grounded, and sensor electrodes, respectively. In Eq. (2), damping is omitted for brevity. The fifth line and column of Eq. (2) are deleted since the electrical potential on the grounded electrodes vanishes. On each potential and sensor electrode, each node has the same electric potential. Thus, the electrical degrees of freedom on the potential and sensor electrodes are combined into master degrees of freedom $\phi_{e p}$ and $\phi_{e m}$, respectively, by applying the transformations

$$
\boldsymbol{\phi}_{p}=\mathbf{T}_{p} \phi_{e p} \text { and } \boldsymbol{\phi}_{m}=\mathbf{T}_{m} \phi_{e m},
$$

with the coincidence transformation matrices $\mathbf{T}_{p}$ and $\mathbf{T}_{m}$. Under the assumption of no structural loads $\mathbf{f}$ and internal charges $\mathbf{q}_{i}$ and by moving the excitation terms of the potential electrodes to the right side, the dynamic equations are written as

$$
\underbrace{\left[\begin{array}{ccc}
\mathbf{M}_{s s} & \mathbf{0} & \mathbf{0} \\
\mathbf{0} & \mathbf{0} & \mathbf{0} \\
\mathbf{0} & \mathbf{0} & \mathbf{0}
\end{array}\right]}_{\mathbf{M}_{\mathrm{EV}}}\left[\begin{array}{c}
\ddot{\mathbf{u}} \\
\ddot{\boldsymbol{\phi}} \\
\ddot{\boldsymbol{\phi}}_{e m}
\end{array}\right]+\underbrace{\left[\begin{array}{ccc}
\mathbf{K}_{s s} & \mathbf{K}_{s i} & \mathbf{K}_{s m} \mathbf{T}_{m} \\
\mathbf{K}_{s i}^{T} & \mathbf{K}_{i i} & \mathbf{K}_{i m} \mathbf{T}_{m} \\
\mathbf{T}_{m}^{T} \mathbf{K}_{s m} & \mathbf{T}_{m}^{T} \mathbf{K}_{i m} & \mathbf{T}_{m}^{T} \mathbf{K}_{m m} \mathbf{T}_{m}
\end{array}\right]}_{\mathbf{K}_{\mathrm{EV}}}\left[\begin{array}{c}
\mathbf{u} \\
\boldsymbol{\phi}_{i} \\
\boldsymbol{\phi}_{e m}
\end{array}\right]=-\underbrace{\left[\begin{array}{c}
\mathbf{K}_{s p} \mathbf{T}_{p} \\
\mathbf{K}_{i p} \mathbf{T}_{p} \\
\mathbf{T}_{m}^{T} \mathbf{K}_{m p} \mathbf{T}_{p}
\end{array}\right]}_{\mathbf{K}_{\mathrm{exc}}} \boldsymbol{\phi}_{e p} .
$$

For harmonic excitation $\phi_{e p}=\phi_{0} e^{j \omega t}$, the corresponding eigenvalue problem is given by

$$
\mathbf{K}_{\mathrm{EV}} \boldsymbol{\psi}_{r}=\omega_{r}^{2} \mathbf{M}_{\mathrm{EV}} \boldsymbol{\psi}_{r},
$$

with the eigenvectors $\boldsymbol{\psi}_{r}$. The system is transformed into modal coordinates with the transformation law $\mathbf{u}=\mathbf{\Psi q}$, where $\boldsymbol{\Psi} \in \mathbb{R}^{n \times m}$ is the modal matrix containing $m \ll n$ low-frequency mode shapes $\boldsymbol{\psi}_{r}, \quad r=1, \ldots, \quad m$, i.e., $\boldsymbol{\Psi}=\left[\boldsymbol{\psi}_{1}, \ldots, \boldsymbol{\psi}_{m}\right]$. Normalization of the modal mass and stiffness matrices results in the identity matrix $\mathbf{I} \in \mathbb{R}^{m \times m}$ and the spectral matrix $\Lambda \in \mathbb{R}^{m \times m}$, respectively, i.e.,

$$
\begin{aligned}
& \mathbf{M}_{m}=\boldsymbol{\Psi}^{T} \mathbf{M}_{\mathrm{EV}} \boldsymbol{\Psi}=\mathbf{I}, \\
& \mathbf{K}_{m}=\boldsymbol{\Psi}^{T} \mathbf{K}_{\mathrm{EV}} \boldsymbol{\Psi}=\boldsymbol{\Lambda}=\operatorname{diag}\left(\omega_{r}^{2}\right) .
\end{aligned}
$$

This reduced system is typically referred to as the normal mode model and is valid only for frequencies well below the highest-frequency eigenmode kept in the truncated transformation matrix. In modal coordinates, the system is expressed as

$$
\mathbf{I} \ddot{\mathbf{q}}+\Lambda \mathbf{q}=-\boldsymbol{\Psi}_{\mathrm{HSV}}^{T} \mathbf{K}_{\mathrm{exc}} \phi_{e p} .
$$

A further reduction of the model order is obtained by selecting those mode shapes of the system which are dominant in terms of controllability and observability. The controllability of a mode specifies its excitation by input actuation, whereas observability describes the influence of the modal states on the system outputs. Controllability and observability are quantified by the controllability Grammians $\mathbf{W}_{c}$ and observability Grammians $\mathbf{W}_{o}$, respectively, which are diagonally dominant for systems in modal representation, ${ }^{21}$ i.e., $\mathbf{W}_{c} \approx \operatorname{diag}\left(w_{c_{i}}\right)$ and $\mathbf{W}_{o} \approx \operatorname{diag}\left(w_{o_{i}}\right)$, where $w_{c_{i}}$ and $w_{o_{i}}$ are the modal controllability and observ- ability coefficients of the $i$ th mode. The transfer function of a structural system is a composition of the modal transfer functions $G_{m_{i}}$ of the modes $i$, i.e., $G(\omega)=\sum_{i=1}^{n} G_{m_{i}}(\omega)$. The modal $H_{\infty}$-norm $\left\|G_{m_{j}}\right\|_{\infty}=2 \gamma_{j}$, where $\gamma_{j}$ denotes the Hankel singular value (HSV) of the $j$ th mode, is used as a measure of contribution of the $j$ th mode to the single-input, single-output (SISO) transfer function $G(\omega)$. Furthermore, for systems with low modal damping ratios (and thus for the special case of piezoelectric tube scanners), the HSVs are approximated by $\gamma_{i}=\sqrt{w_{c_{i}} w_{o_{i}}{ }^{2}}{ }^{21}$ Modes with low HSVs and thus low modal norms as well as low controllability and observability are not taken into account in the reduction basis $\boldsymbol{\Psi}$ of Eqs. (6) and (7), resulting in the transformation basis $\boldsymbol{\Psi}_{\mathrm{HSV}} \in \mathbb{R}^{n \times m_{\mathrm{HSV}}}$. The fully reduced system is now obtained by replacing $\boldsymbol{\Psi}$ with the truncated transformation matrix $\boldsymbol{\Psi}_{\mathrm{HSV}}$ in Eqs. (6) and (7).

A calculation of the HSVs for the piezoelectric tube scanner reveals that the torsional mode with the eigenvector $\psi_{5}$ has much lower influence on the input-output behavior compared to other low-frequency modes. Thus, the torsional mode shape $\psi_{5}$ is neglected in the transformation basis $\boldsymbol{\Psi}_{\mathrm{HSV}}$. Of each pair of the first three longitudinal bending modes and first two circumferential bending modes, one eigenvector, which is rotated to point along the $x$-direction, is retained in the transformation matrix. Furthermore, the longitudinal extension $\psi_{6}$ is taken into account. Overall, the truncation basis is given by $\boldsymbol{\Psi}_{\mathrm{HSV}}$ $=\left[\boldsymbol{\psi}_{1}, \boldsymbol{\psi}_{3}, \boldsymbol{\psi}_{6}, \boldsymbol{\psi}_{7}, \boldsymbol{\psi}_{9}, \boldsymbol{\psi}_{11}\right]$, leading to a system order $m=6$ in the second order system of Eq. (8), compared to $n \approx 10000$ in Eq. (1). By this truncation, the reduced order model is valid in the frequency range up to approximately $15 \mathrm{kHz}$.

For controller design purposes, the reduced system is 

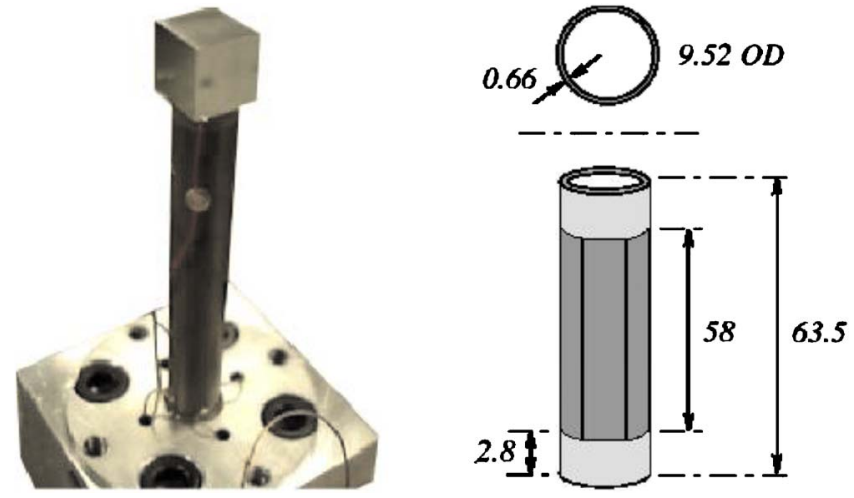

FIG. 4. (Color online) Measurement setup of tube actuator with sample holder glued into an aluminum base and tube dimensions in millimeters.

finally transformed into the modal state space representation obtained form Eq. (8) as

$$
\begin{aligned}
& \dot{\mathbf{x}}=\underbrace{\left[\begin{array}{cc}
\mathbf{0} & \mathbf{I} \\
-\boldsymbol{\Lambda} & -\boldsymbol{\Gamma}
\end{array}\right]}_{\mathbf{A}_{\mathrm{red}}} \mathbf{\mathbf { x }}+\underbrace{\left[\begin{array}{c}
\mathbf{0} \\
-\boldsymbol{\Psi}_{\mathrm{HSV}}^{T} \mathbf{K}_{\mathrm{exc}}
\end{array}\right]}_{\mathbf{B}_{\mathrm{red}}} \mathbf{u}, \\
& \mathbf{y}=\underbrace{\left[\mathbf{c} \boldsymbol{\Psi}_{\mathrm{HSV}} \mathbf{0}\right.}_{\mathbf{C}_{\mathrm{red}}}] \mathbf{x},
\end{aligned}
$$

with the state space vector $\mathbf{x}=\left[\mathbf{q}^{T}, \dot{\mathbf{q}}^{T}\right]^{T}$ and the input vector $\mathbf{u}=\left[\phi_{e p}\right]^{T}$. The output shape matrix $\mathbf{c}$ defines the desired degrees of freedom for the output $y$, which is given in physical nodal quantities. The damping matrix $\boldsymbol{\Gamma}$ is generally derived from the Rayleigh damping of the FEA with typical values of piezoelectric materials for the damping coefficients. If measurement results are available for specific tubes, the damping matrix $\boldsymbol{\Gamma}$ can be easily changed to account for modal damping with the damping coefficients identified from experimental data. Thus, a model with an accurate description of the output displacement is obtained.

\section{MEASUREMENT SETUP}

The piezoelectric tube scanner, shown in Fig. 4 (Boston PiezoOptics, manufactured from high-density PZT-5H), is glued into a recessed aluminum base, which is mounted onto an optical table. On the outer diameter, four equally spaced silver electrodes are deposited in quadrature. Geometric dimensions of the tube are also given in Fig. 4.

A Polytec PI PSV300 laser doppler vibrometer is used to measure the tip displacement frequency response by excitation of one $x$-electrode with a $5.5 \mathrm{~V}$ periodic chirp signal with a bandwidth of $25 \mathrm{kHz}$. The measured signals were sampled at $52.1 \mathrm{kHz}$ and a 6400 line fast Fourier transform (FFT) is computed to obtain the frequency response functions.

\section{RESULTS AND DISCUSSION}

The simulations and experiments are carried out for antisymmetric (single electrode) excitation. The frequency response functions (FRFs) describe the $x$-, $y$-, and $z$-displacements at the tip of the scanner-sample unit, i.e., at the top of the sample holder, denoted by $u_{x}, u_{y}$, and $u_{z}$, and, in addition, the induced voltages at the $x$-and $y$-axis sensor electrodes, $m v_{x}$ and $m v_{y}$, respectively. The system of Eq. (9) is thus single-input multiple-output, with the scalar input $\mathbf{u}$ $=v_{x}$ denoting the driving voltage at the $x$-axis potential electrode and the output vector $\mathbf{y}=\left[u_{x}, u_{y}, u_{z}, m v_{x}, m v_{y}\right]^{T}$. In case of the reduced model, the calculation time for the FRFs drops to some seconds compared to approximately one hour necessary to carry out the harmonic analysis of the full FE model. However, note that the model reduction is of special importance for the design of sophisticated controllers, ${ }^{23,24}$ where optimal controllers are typically of the same order as the plant. ${ }^{25}$ The frequency responses of the experimental system together with the full and reduced order FE models are shown in Figs. 5-9. First, the dynamics resulting from the nominal scan unit consisting of tube and sample holder are considered. The effects of deviations from the nominal model, i.e., from tube eccentricity and asymmetric electrode placement, are then investigated by simulation studies. All measured and computed FRFs include the sample mass at the free end of the tube.

\section{A. Nominal model}

The FRFs for the $x$-axis displacement in Fig. 5 for the full and low order FE models show an excellent agreement to the measured data over a wide frequency range. This response is strongly dominated by the three low-frequency lon-
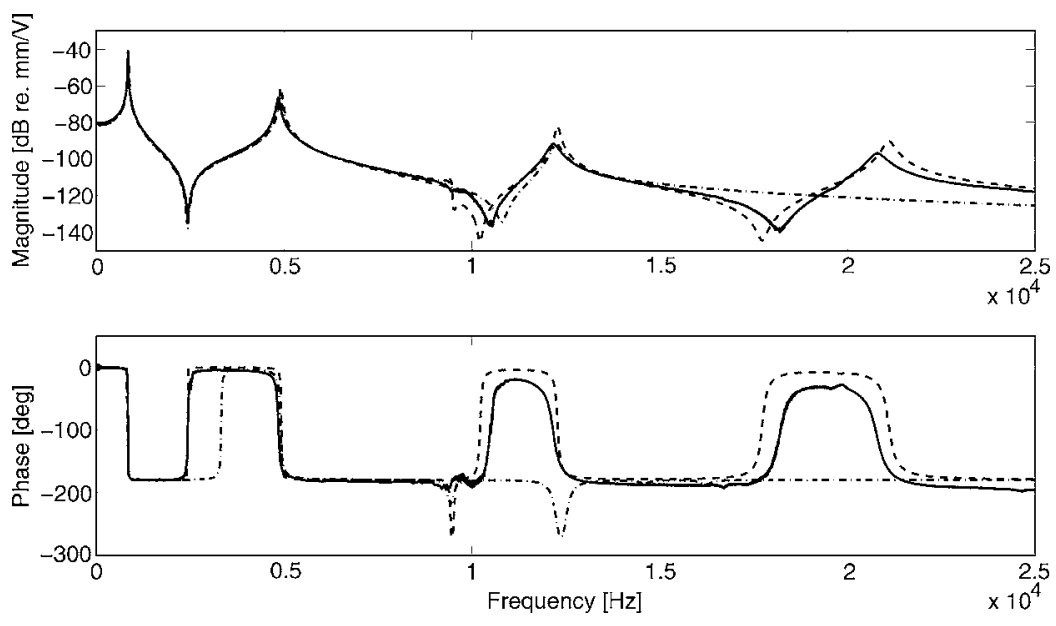

FIG. 5. Comparison of FRFs for $x$-displacement of measurement (solid curve), full finite element model (dashed curve), and reduced low order model (dasheddotted curve) for single electrode excitation. 

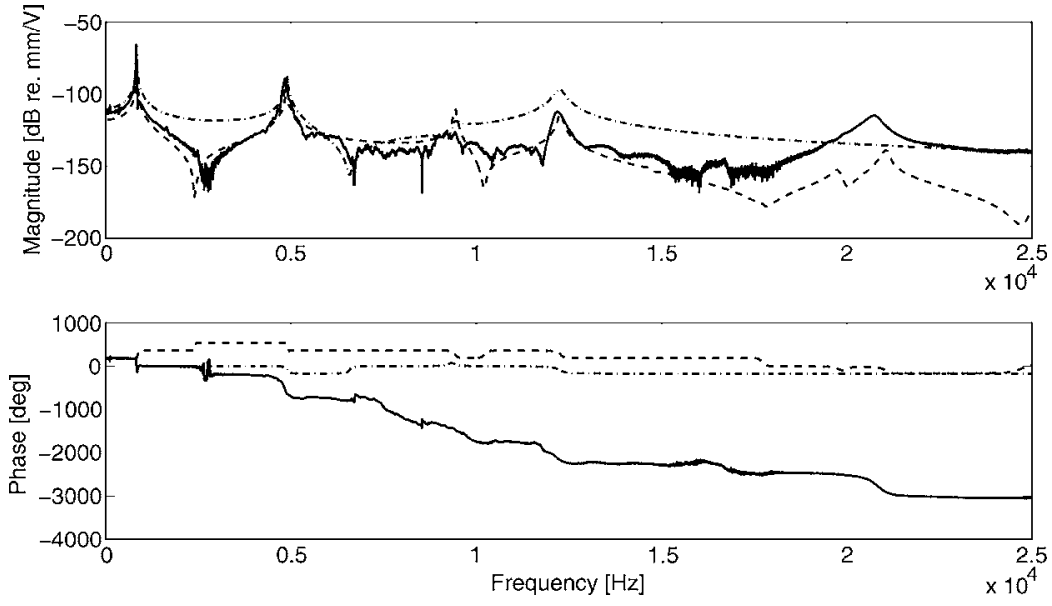

FIG. 6. Comparison of FRFs for $y$-displacement of measurement (solid curve), full finite element model (dashed curve), and reduced low order model (dasheddotted curve) for single electrode excitation. gitudinal bending modes since the excitation at the driving electrode and the $x$-displacement output point in the same direction. The good agreement for the longitudinal bending modes is obvious from the almost perfectly matching eigenfrequencies in Table II and is demonstrated in Ref. 7 for the corresponding mode shapes. Note that by switching the damping model from Rayleigh damping with standard parameters of piezoelectric materials to modal damping in the reduced model, it is possible to accurately match the resonance amplitudes in the simulation data, e.g., for the third longitudinal bending mode at approximately $12 \mathrm{kHz}$ in Fig. 5.

For the $y$-axis displacement in Fig. 6, the FE model is in accordance with the experimental amplitude response. At high frequencies, the model underestimates the measured response but reflects the main measured resonance peaks. However, the amplitude and phase response of the reduced model considerably deviates from the full model, which can be explained by the low order, where only one eigenvector of a pair of the bending mode shapes is contained in the truncation basis. This single eigenvector does not completely account for the directional coupling between the $x$-axis excitation and $y$-axis displacement output. The order of the HSV of the eigenmodes in Sec. II B is arranged by mainly weighting $x$ - and $z$-axis displacement output behaviors. A reduction basis composed of all low-frequency bending mode shapes, i.e., the corresponding pairs, accurately retains FE-model dynamics even for the $y$-displacement (data not shown). The displacement coupling from the $x$-voltage excitation to the $y$-displacement output amounts to $3.16 \%$ of the $x$-displacement in the static limit, i.e., for a desired $x$-displacement of $1 \mu \mathrm{m}$, the $y$-displacement from coupling results in $0.03 \mu \mathrm{m}$. This is a considerable deviation from the desired trajectory and should be compensated, e.g., by feedforward or feedback techniques. The coupling in the lateral plane is caused by breaking the symmetry of the tube geometry in the setup considered, where one $y$-electrode is grounded while the other one is used as a sensor electrode. This leads to an unequal charge distribution on these electrodes, which in turn results in an undesired bending moment along the $y$-axis. The phase mismatch of the FE models to the measurement in Fig. 6 is associated to the smooth phase decay in the experiment, such that the phase cannot be restricted to a $360^{\circ}$ range in contrast to the simulation.

The modeled displacements along the $z$-axis in Fig. 7 are consistent with the measured amplitude response. The model overestimates the static displacement and the influence of the second longitudinal bending mode but accurately describes the dominance of the longitudinal extension mode shape at approximately $9 \mathrm{kHz}$. For the $x$ to $z$ coupling, the $z$-displacement constitutes about $17.7 \%$ of the $x$-displacement in the simulation data and approximately $5.6 \%$ in the measured response.

The induced voltages in Figs. 8 and 9 are of interest for
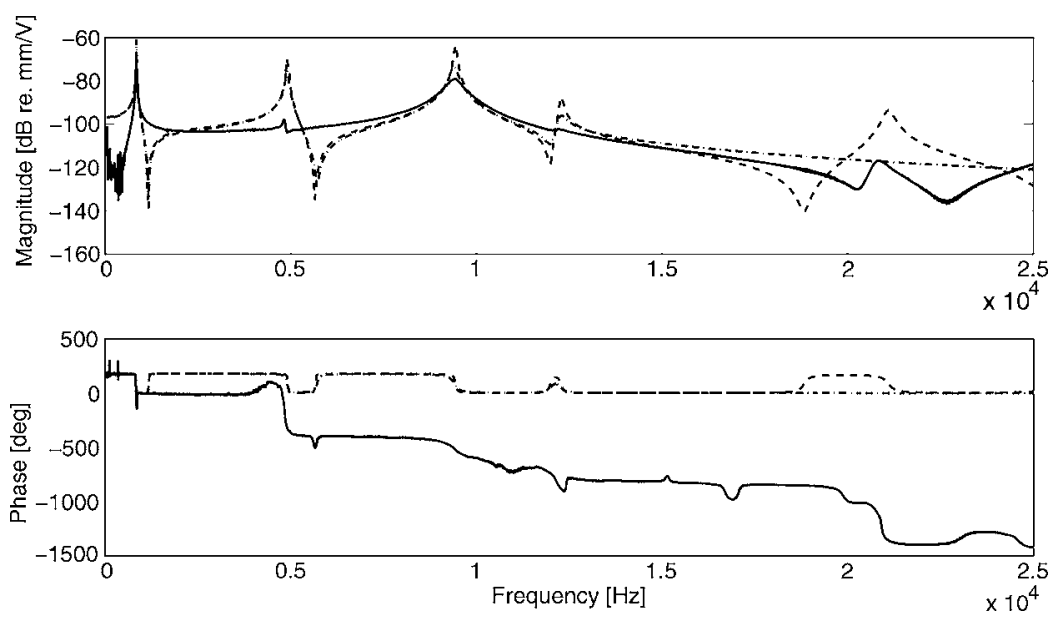

FIG. 7. Comparison of FRFs for $z$-displacement of measurement (solid curve), full finite element model (dashed curve), and reduced low order model (dasheddotted curve) for single electrode excitation. 

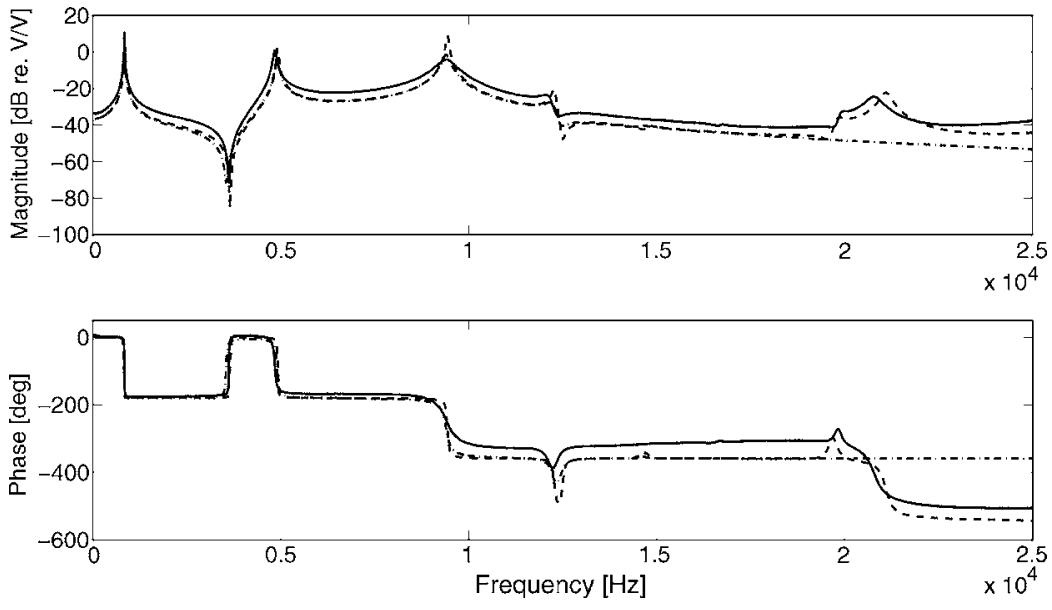

FIG. 8. Comparison of FRFs for induced voltage on $x$-electrode of measurement (solid curve), full finite element model (dashed curve), and reduced low order model (dashed-dotted curve) for single electrode excitation. closed-loop control implementation. In the standard operation of piezoelectric tube scanners, the measurement of the tube displacement requires additional sensors. This drawback is circumvented by employing the induced voltages at the sensor electrodes as feedback signals. Voltage measurement does not require additional sensors and is thus relatively simple to implement. The FE-modeled induced voltages at the $x$-axis sensor electrode in Fig. 8 and the $y$-axis sensor electrode in Fig. 9 both accurately match the measurement outputs over the whole frequency range. The reduced order model also shows good agreement with the experimental results but there appears to be a mismatch of a constant gain compared to the FE model. This might be due to the existence of a feedthrough component for sensor voltages in the model reduction process ${ }^{26}$ and is the subject of ongoing research. Note that the longitudinal extension mode is more pronounced in the sensed voltages compared to the corresponding displacement outputs, leading to significantly stronger coupling between $x$ - and $y$-motions for the induced voltages. It is pointed out that single electrode excitation to enable induced voltage measurement at the sensor electrode reduces the scan range by half compared to twin electrode excitation.

\section{B. Simulation study of geometrical variations}

With respect to controller robustness, the closed-loop operation of piezoelectric tube scanners is affected by plant uncertainties. These uncertainties are partially due to machining imperfections in the manufacturing process of the piezoelectric tube. Common problems are the concentricity of the tube geometry and the exact location and shape of the deposited electrodes on the outer tube diameter. The generality of the FE method allows the investigation of these deviations by simulation studies. Furthermore, the effect of shortened electrodes on piezotube dynamics is analyzed, which is of interest for gaining space towards additional actuator or sensor electrode placement, e.g., for distinct $z$-displacement actuation.

The simulation data for several variations of the tube geometry on the dynamics-coupling between $x$-axis excitation and $y$-axis displacement response is shown in Figs. 10-12. In Fig. 10, the nominal tube is compared to a setup in which both $y$-electrodes are grounded to restore a symmetrical charge distribution with respect to the $y$-axis, while the tube geometry and electrode locations are conserved. Obviously, the dynamics-coupling effects in the $y$-direction are drastically reduced by this measure, especially for low frequencies.

The $y$-displacement coupling for an eccentric tube is illustrated in Fig. 11 for eccentricities of $50 \mu \mathrm{m}$ in the $x$ - and $y$-axes, respectively. Compared to the nominal model, the coupling is amplified for all frequencies. Similar results are obtained for a concentric tube geometry, but with the potential electrode radially shifted by $15^{\circ}$ plotted in Fig. 12. In
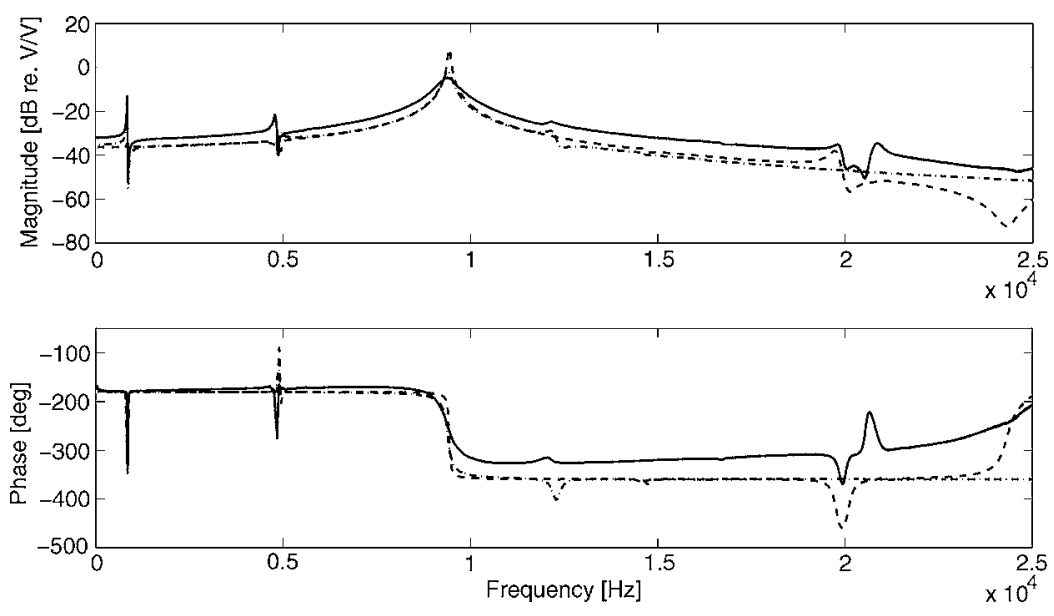

FIG. 9. Comparison of FRFs for induced voltage on $y$-electrode of measurement (solid curve), full finite element model (dashed curve), and reduced low order model (dashed-dotted curve) for single electrode excitation. 


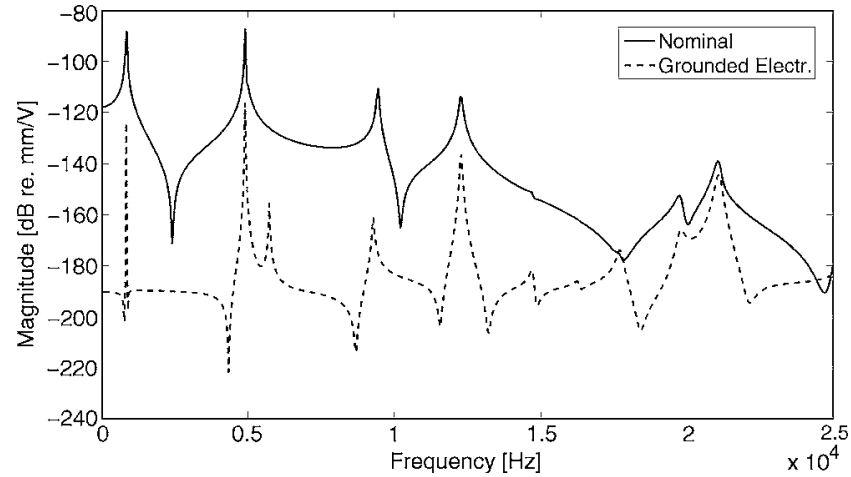

FIG. 10. Simulation of coupling form $x$-excitation to $y$-displacement for both $y$-electrodes grounded.

summary, every change to the scan unit, which breaks either the geometrical or the electric field symmetry with respect to the $y$-axis leads to an increase in lateral dynamics coupling.

Figure 13 describes the dynamics in the case of shortened electrodes, which cover only half of the axial length of the piezoelectric tube. The $x$-axis displacement output in Fig. 13 is reduced to approximately $68 \%$ compared to the full electrode length in the steady-state limit. This demonstrates, that the scan range of the scanner is rigorously influenced by the electrode length.

In summary, the main contributions of the measurement and simulation are split into the following observations: (1) the FEA gives a precise description of the piezoscanner dynamics with sample mass; (2) the induced voltages can be employed as feedback signals; (3) dynamics-coupling effects are mainly due to the electrode circuitry, tube eccentricity, and dislocated electrodes; and (4) electrode size and shape significantly changes the scanner dynamics and scan range.

\section{CONCLUSIONS}

Different aspects of the dynamic behavior of piezoelectric tube actuators are investigated by FE-model simulation carried out in ANSYS. Measurement results for a tube scanner with sample holder attached to its free end demonstrate the high accuracy of FEA. Furthermore, a model order reduction scheme, which keeps the nodal displacements as well as the induced sensor electrode voltages as signal outputs, is presented. The reduced model is therefore suited for

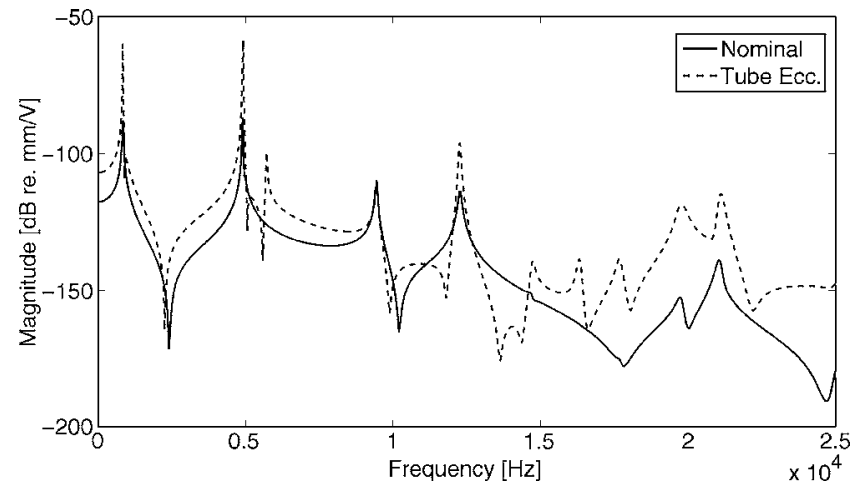

FIG. 11. Simulation of coupling form $x$-excitation to $y$-displacement for eccentric tube.

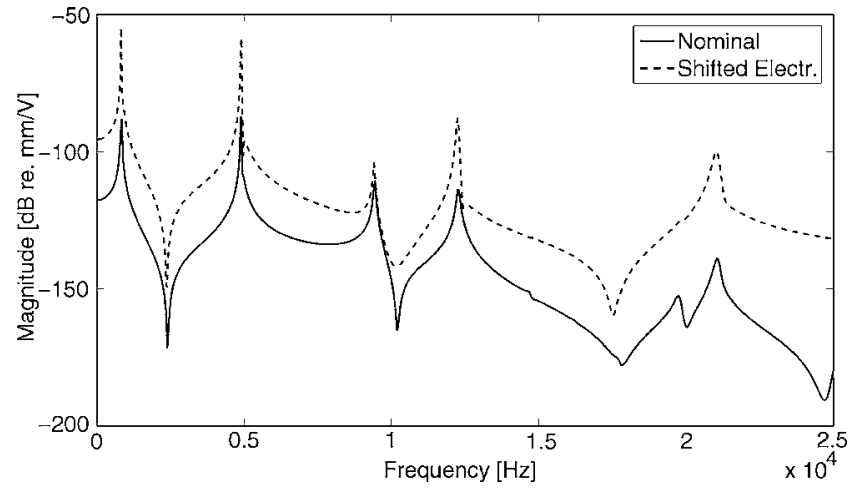

FIG. 12. Simulation of coupling form $x$-excitation to $y$-displacement for shifted potential electrode.

model-based feedforward and feedback controller designs, where an appropriate output signal which does not require additional displacement sensors is made available by means of the induced voltages. The foremost benefits of this modeling approach are the possibility to model variations in the piezoscanner setup (i.e., sample holder, tube eccentricity, and electrode location/shape), and the flexibility in the model order reduction to obtain either single-input single-output (SISO) or multi-input multi-output (MIMO) systems. Owing to the ability of the model to realistically describe common tube imperfections and dynamic variations, e.g., from varying sample mass, it is suited for the analysis of the robustness of feedforward and feedback controllers. In particular, the models capture all dynamics-coupling effects, with results presented for voltage excitation along the $x$-axis and displacement outputs in $y$ - and $z$-directions, enabling a model-based compensation of coupling-caused errors in the scan trajectory.

The main reason for dynamics-coupling effects in the lateral plane is identified by simulation results as a breaking of the tube symmetry relative to the $x$-or $y$-axis. It is shown that this asymmetric configuration can either be caused mechanically by tube eccentricity or in the electric field distribution by dislocated electrodes or by nonuniformly circuited electrodes. The generality of the model is furthermore used to investigate the dynamic behavior of the tube for shortened electrodes, where a significant reduction in scan range is observed. It is possible that the FE model could be used for the optimization of the shape of the actuator and sensor elec-

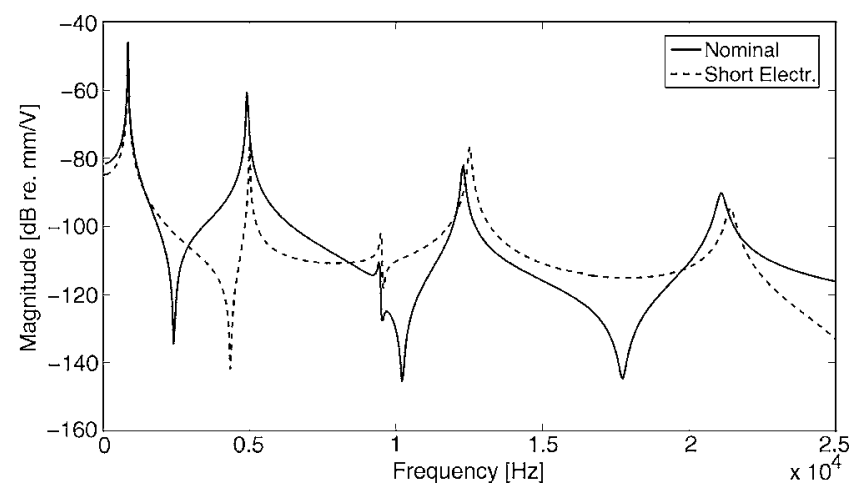

FIG. 13. Simulation of $x$-displacement for shortened electrodes. 
trodes towards a reduction of dynamics coupling and thus increased scanning precision. This is the subject of present research.

\section{ACKNOWLEDGMENTS}

Experimental work was performed at the Laboratory for Dynamics and Control of Nanosystems, The University of Newcastle.

${ }^{1}$ A. J. Fleming and S. O. R. Moheimani, IEEE Trans. Control Syst. Technol. 14, 33 (2006).

${ }^{2}$ G. Binning and D. P. E. Smith, Rev. Sci. Instrum. 57, 1688 (1986).

${ }^{3}$ M.-S. Tsai and J.-S. Chen, J. Dyn. Syst., Meas., Control 125, 96 (2003).

${ }^{4}$ O. M. El Rifai and K. Youcef-Toumi, Proceedings of the American Control Conference (Anchorage, AK, 2002), p. 3777.

${ }^{5}$ S. Tien, Q. Zou, and S. Devasia, IEEE Trans. Control Syst. Technol. 13, 921 (2005)

${ }^{6}$ D. Croft, G. Shed, and S. Devasia, J. Dyn. Syst., Meas., Control 123, 35 (2001).

${ }^{7}$ J. Maess, A. J. Fleming, and F. Allgöwer, Proceedings of the American Control Conference (New York, NY, 2007), p. 4221.

${ }^{8}$ O. M. El Rifai and K. Youcef-Toumi, Proceedings of the American Control Conference (Arlington, VA, 2001), p. 3251.

${ }^{9}$ U. Gabbert, T. N. Trajkov, and H. Köppe, Facta Univers. 3, 417 (2002).

${ }^{10}$ G. Song, J. Zhao, X. Zhou, and J. A. De Abreu-García, IEEE/ASME Trans. Mechatron. 10(2), 198 (2005).
${ }^{11} \mathrm{Y}$. Wu and Q. Zou, Proceedings of the American Control Conference (Minneapolis, MN, 2006), p. 424.

${ }^{12}$ K. El Rifai, O. El Rifai, and K. Youcef-Toumi, Proceedings of the American Control Conference (Boston, MA, 2004), p. 3128.

${ }^{13}$ S.-H. Chao, J. L. Garbini, W. M. Dougherty, and J. A. Sidles, Rev. Sci. Instrum. 77, 063710 (2006).

${ }^{14}$ A. Benjeddou, M. A. Trindade, and R. Ohayon, J. Intell. Mater. Syst. Struct. 8, 1012 (1997)

${ }^{15}$ Y.-H. Lim, Smart Mater. Struct. 12, 272 (2003).

${ }^{16}$ R. G. Carr, J. Microsc. 152, 379 (1988).

${ }^{17}$ Q. Sun and R. A. Wolkow, Rev. Sci. Instrum. 77, 113701 (2006).

${ }^{18}$ M. Ratnam, B. Bhikkaji, A. J. Fleming, and S. O. R. Moheimani, Proceedings of the Conference on Decision and Control (Sevilla, Spain, 2005) p. 1168.

${ }^{19}$ A. Preumont, Vibration Control of Active Structures (Kluwer, Dordrecht, 1997).

${ }^{20}$ ANSYS, Inc., ANSYS Documentation 10.0 (2004).

${ }^{21}$ W. K. Gawronski, Advanced Structural Dynamics and Active Control of Structures (Springer, New York, 2004).

${ }^{22}$ J. Becker, O. Fein, M. Maess, and L. Gaul, Comput. Struct. 84, 2340 (2006).

${ }^{23}$ S. Salapaka, A. Sebastian, J. P. Cleveland, and M. V. Salapaka, Rev. Sci. Instrum. 73, 3232 (2002).

${ }^{24}$ G. Schitter, F. Allgöwer, and A. Stemmer, Nanotechnology 15, 108 (2004).

${ }^{25}$ S. Skogestad and I. Postlethwaite, Multivariable Feedback Control (Wiley, Chichester, 2005).

${ }^{26}$ V. Piefort, "Finite Element Modelling of Piezoelectric Active Structures," Ph.D. thesis, Université Libre de Bruxelles, 2001. 